\title{
Liquid-immersion laser micromachining of GaN grown on sapphire
}

\author{
Giuseppe Y. Mak · Edmund Y. Lam $\cdot$ H.W. Choi
}

Received: 28 October 2010 / Accepted: 9 December 2010 / Published online: 22 December 2010

(c) The Author(s) 2010. This article is published with open access at Springerlink.com

\begin{abstract}
Liquid-immersion nanosecond-pulsed laser micromachining is introduced as an efficient way for device isolation and rapid prototyping on $\mathrm{GaN}$-on-sapphire wafer. Using deionized water as an ambient medium for laser micromachining, smooth trenches that are free from redeposition can be formed in the GaN layer. Coupled with the large difference between the ablation thresholds and ultraviolet absorption coefficients of GaN and sapphire, the $\mathrm{GaN} /$ sapphire interface can be left undamaged after the ablation process. This technique overcomes the limitation of heat accumulation in nanosecond-pulse regime, and offers a cost-effective alternative to ultrashort-pulse laser micromachining. In this report, the advantages offered by liquid immersion are elucidated in terms of improved heat conduction, increased plasma-induced recoil pressure due to water confinement, weakened plasma shielding effect in water, and the collapse of cavitation bubbles. Simulation results show that the reduced fluctuation of temperature profile over time in water could be correlated with the reduced redeposition of $\mathrm{Ga}$ from thermal decomposition at the trench sidewalls.
\end{abstract}

\section{Introduction}

Ultraviolet (UV) pulsed laser has been a powerful tool for the dicing [1] and micromachining [2] of GaN-on-sapphire light-emitting diode (LED) wafer. Laser beams with sufficient power density are able to ablate $\mathrm{GaN}$ and/or sapphire, producing micro- or sub-micron features such as holes

G.Y. Mak · E.Y. Lam · H.W. Choi ( $\varangle)$

Semiconductor Lighting and Display Laboratory, The University of Hong Kong, Pokfulam Road, Hong Kong, Hong Kong

e-mail: hwchoi@hku.hk and trenches on the wafer. A combination of these features can form roughened surfaces [3] and mesa structures with tapered sidewalls [4] for improving light extraction and achieving device isolation. In order to attain good surface morphology and reduce the amount of redeposition after laser ablation, laser parameters such as pulse duration, laser fluence and pulse repetition rate have to be optimized. Since the pulse duration of nanosecond lasers is long enough to cause thermal damage, recent research effort has been directed to the application of ultrashort-pulsed lasers (in the range of picoseconds to femtoseconds) for non-thermal ablation, for example, through multiphoton absorption mechanism [5]. However, ultrashort-pulsed lasers are not without shortcomings. High cost, bulkiness, and the difficulty in aligning the pumping source, gain medium and frequency doubling crystal limit their widespread use. As nanosecondpulsed laser is currently the prevalent technology in the LED industry, it is still desirable to develop improved methods to overcome its limitations.

Previously, the authors have reported on a nanosecond-laser micromachining technique for the formation of trenches in epitaxially-grown $\mathrm{GaN}$ on c-plane sapphire substrate [6]. The major characteristic of this technique is the "auto-stop" ablation mechanism. When the laser fluence is within the optimum range, the ablation can terminate automatically at the GaN/sapphire interface without damaging the sapphire. This is possible since the two materials are very different in terms of the following two parameters: (1) ablation threshold $\left(0.25 \mathrm{~J} / \mathrm{cm}^{2}\right.$ for GaN [7] vs. $4.5 \mathrm{~J} / \mathrm{cm}^{2}$ for sapphire [8]), and (2) UV absorption coefficient $\left(1.0-1.5 \times 10^{5} \mathrm{~cm}^{-1}\right.$ for GaN [9] vs. $0.01-1 \mathrm{~cm}^{-1}$ for sapphire [10]). The technique is particularly suitable for device isolation and rapid prototyping of LED chips. Nevertheless, even at the optimum laser fluence, substantial amount of redeposition and resolidification of ablated material still 
exists on the trench bottom surface and sidewall. In order to reduce the heat load during ablation, we propose performing the micromachining process in a liquid ambient. Such liquid must have good thermal conductivity and high specific heat capacity. In addition, the attenuation of UV and visible light in that liquid should be low, so that laser energy can be transferred efficiently to the substrate and the micromachining process can be monitored concurrently; deionized (DI) water would be a good choice. Underwater laser drilling on glass and metal plates has been studied by a number of researchers [11-13]. The mechanisms involved in liquidimmersion laser micromachining of $\mathrm{GaN}$ are explained in this work.

\section{Experimental setup}

The characteristics of liquid-immersion laser micromachining for $\mathrm{GaN}$ were investigated experimentally using the optical setup shown in Fig. 1. The collimated third-harmonic $\mathrm{Nd}$ :YLF laser beam $(\lambda=349 \mathrm{~nm}$, full-width half-maximum (FWHM) pulse width $\tau_{\mathrm{FWHM}}=4 \mathrm{~ns}$, pulse repetition rate $=$ $1 \mathrm{kHz})$ was focused by a UV objective lens $(f=19 \mathrm{~mm})$. The sample was immersed horizontally in a DI water bath with meniscus about $1 \mathrm{~mm}$ above the sample surface. Although thicker water layer can improve heat dissipation, attenuation of the laser beam will become more severe. On the other hand, a water layer thinner than $1 \mathrm{~mm}$ is not possible due to the strong surface tension of water. The wafer consisted of a 3- $\mu \mathrm{m}$ thick GaN layer (with $\mathrm{p}-\mathrm{GaN}$, InGaN/GaN multi-quantum well (MQW) and $n-G a N)$ and a $300-\mu \mathrm{m}$ thick sapphire layer. The water bath was placed on a manual $\mathrm{Z}$ translation stage, mounted on a motorized $\mathrm{XY}$ translation stage to enable laser scanning. The laser fluence $\Phi$ was adjusted by offsetting the sample surface from the best focus plane, as shown in Fig. 2, so that the laser spot area is altered. When the focus offset is too small (such that $\Phi \geq 4.5 \mathrm{~J} / \mathrm{cm}^{2}$ ) or too large (such that $\Phi<0.25 \mathrm{~J} / \mathrm{cm}^{2}$ ), either damage to the sapphire layer or incomplete removal of $\mathrm{GaN}$ will be resulted. The optimum focus offset is achieved when $0.25 \mathrm{~J} / \mathrm{cm}^{2} \leq \Phi<4.5 \mathrm{~J} / \mathrm{cm}^{2}$. The measurement of trench surface roughness was performed by atomic force microscopy (AFM) (Seiko Instruments Nanopics 2100), while the surface morphology was imaged by field-emission scanning electron microscope (FE-SEM) (Hitachi S-4800). Elemental analysis of the trench surface was performed by the energy-dispersive X-ray spectroscopy (EDX) function offered by the FE-SEM.

\section{Experimental results}

Compared with laser micromachining in ambient air, DI water is capable of producing trenches with substantially smoother sidewalls and bottom surfaces. Figure 3 shows

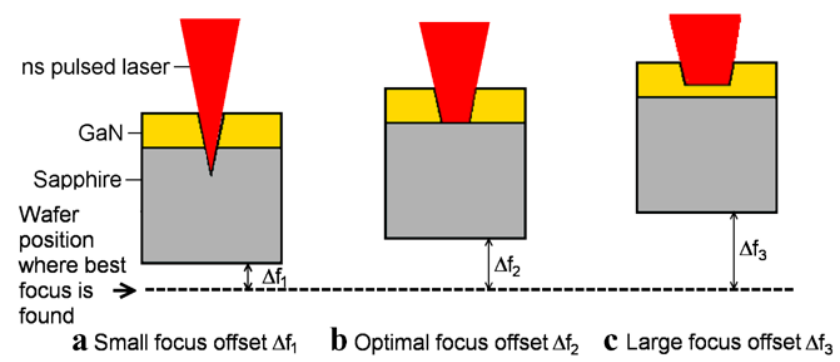

Fig. 2 Laser fluence controlled by focus offset
Fig. 1 Optical setup for liquid-immersion laser micromachining, with the inset showing the meniscus about $1 \mathrm{~mm}$ above the sample

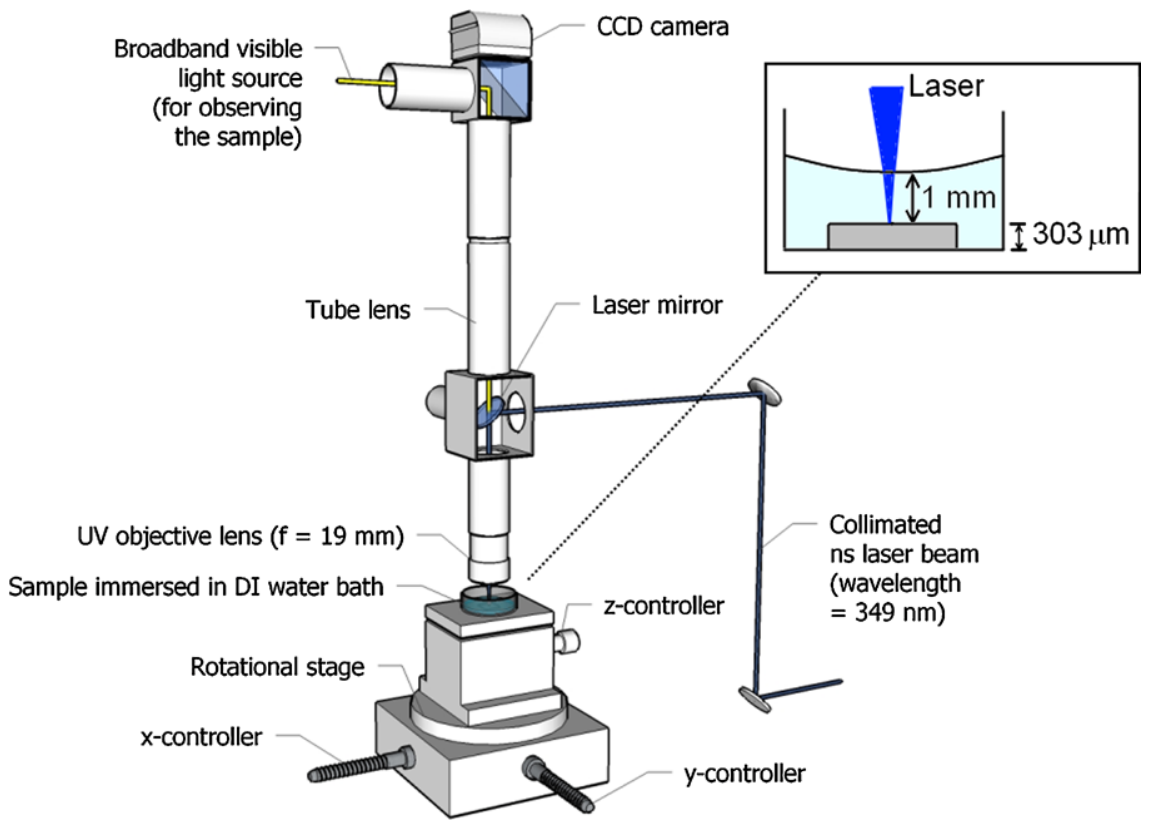




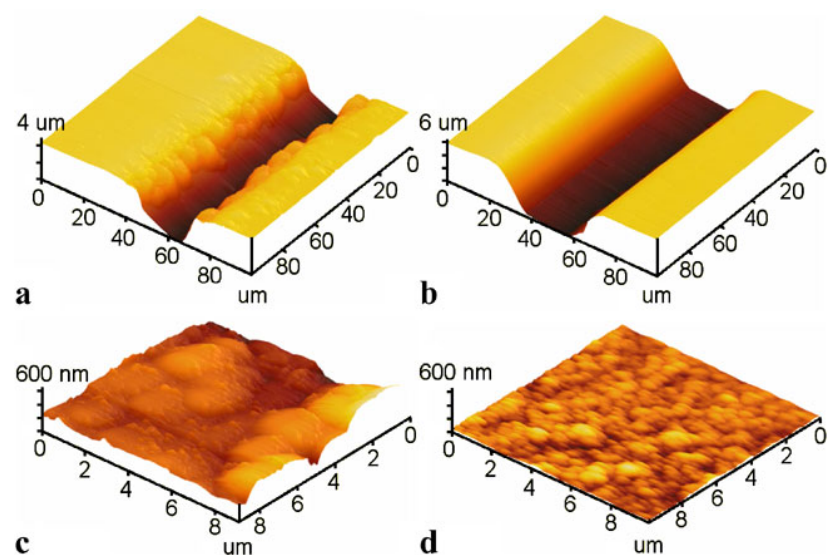

Fig. 3 AFM scans of trenches generated in: (a) and (c) air; (b) and (d) DI water. (c) and (d) are the zoomed-in images of the bottom surfaces. The focus offset, pulse energy, pulse repetition rate and scan speed were fixed at $400 \mu \mathrm{m}, 25 \mu \mathrm{J}, 1 \mathrm{kHz}$ and $25 \mu \mathrm{m} / \mathrm{s}$

AFM scans of the trenches generated in both ambient media. By measuring the height values $y_{i}$ along the trench edge, the sidewall roughness $R_{a}$ is determined by taking the arithmetic average of the absolute height deviation from the mean height $\bar{y}$ :

$R_{a}=\frac{1}{n} \sum_{i=1}^{n}\left|y_{i}-\bar{y}\right|$.

The trench micromachined in air has an $R_{a}$ of $312 \mathrm{~nm}$, contrasting sharply with $R_{a}=27.65 \mathrm{~nm}$ for the trench produced in DI water. The rms roughness of the bottom surfaces also reveals the superiority of liquid immersion $(87.49 \mathrm{~nm}$ for air vs. $13.42 \mathrm{~nm}$ for DI water). According to [14], the rms roughness for the trenches micromachined by femtosecond laser in poly (methyl methacrylate) (PMMA) in ambient air are in the range of $17 \mathrm{~nm}-785 \mathrm{~nm}$. Although the materials involved are different, these data serve as a reference, indicating that nanosecond-laser micromachining in DI water can have comparable performance with femtosecond-laser micromachining in air.

The reduced roughness is partly due to reduced redeposition of ablated material. From the results of EDX (Table 1), three elements were found over the bottom surface of the trenches, namely $\mathrm{O}, \mathrm{Al}$ and $\mathrm{Ga}$. $\mathrm{Al}$ and $\mathrm{O}$ are the constituent elements of sapphire $\left(\mathrm{Al}_{2} \mathrm{O}_{3}\right)$, whereas $\mathrm{Ga}$ is a product from the thermal decomposition of $\mathrm{GaN}$ during laser ablation [15, 16]. It is found that there is a lower percentage of $\mathrm{Ga}$ over those trenches produced in DI water. It should be noted that, while the sample for air had been sonicated in dilute $\mathrm{HCl}$ (18\%) before EDX examination, that for DI water was not subjected to the same treatment. The results indicate that liquid immersion is effective in ejecting the molten $\mathrm{Ga}$ (m.p. = $29.76^{\circ} \mathrm{C}$, b.p. $=2204^{\circ} \mathrm{C}$ ) from the irradiated site and preventing its resolidification around the trench.
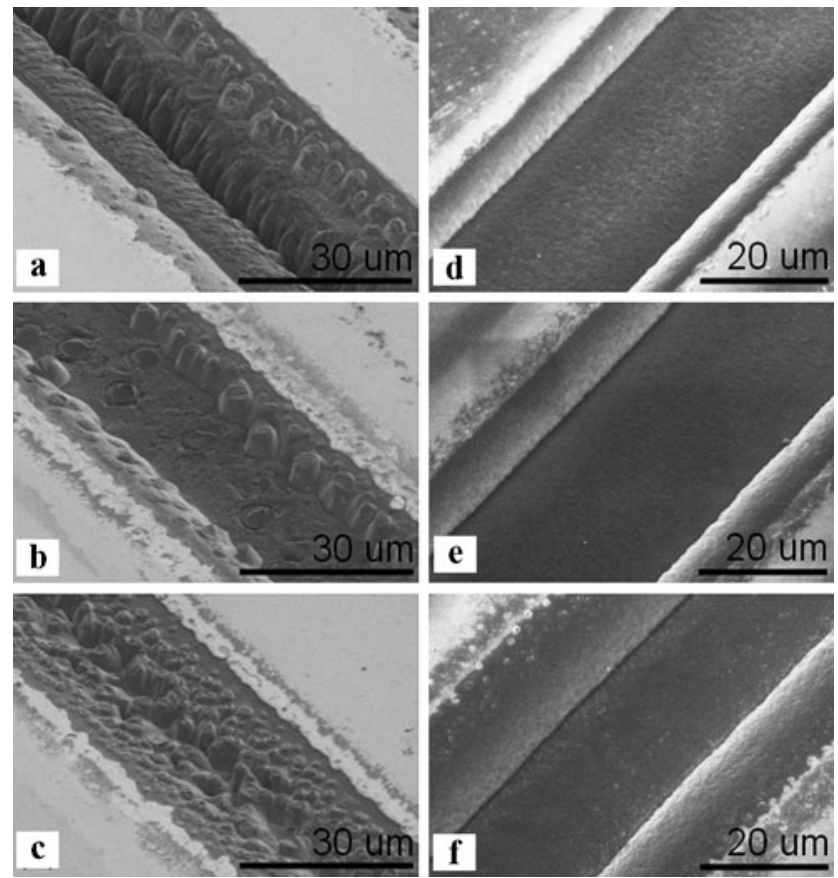

Fig. 4 (a)-(c): SEM images of trenches laser-micromachined at different focus offset planes in air: (a) $300 \mu \mathrm{m}$; (b) $450 \mu \mathrm{m}$; (c) $600 \mu \mathrm{m}$, where $450 \mu \mathrm{m}$ is the optimum focus offset. (d)-(f): SEM images of trenches laser-micromachined at different focus offset planes in DI water: (d) $300 \mu \mathrm{m}$; (e) $500 \mu \mathrm{m}$; (f) $700 \mu \mathrm{m}$. Smooth trenches were observed in all three cases. The pulse energy, pulse repetition rate and scan speed were fixed at $25 \mu \mathrm{J}, 1 \mathrm{kHz}$ and $25 \mu \mathrm{m} / \mathrm{s}$

Table 1 Atomic composition of the bottom surface of trenches

\begin{tabular}{lrr}
\hline & \multicolumn{1}{l}{ Air } & DI water \\
\hline $\mathrm{O}$ & $64.67 \%$ & $65.19 \%$ \\
$\mathrm{Al}$ & $34.81 \%$ & $34.65 \%$ \\
$\mathrm{Ga}$ & $0.52 \%$ & $0.16 \%$ \\
\hline
\end{tabular}

Besides improved surface quality, liquid-immersion laser micromachining also offers two advantages with respect to process control. The first is increased focus offset tolerance. Figures 4(a)-(c) show three trenches micromachined in ambient air. When the focus offset deviates from the optimum plane (Fig. 4(b)) by $150 \mu \mathrm{m}$, either damage to the sapphire layer due to overablation (Fig. 4(a)) or incomplete trench due to underablation of GaN (Fig. 4(c)) occurs. For micromachining in DI water, the trench quality is not compromised even with a focus offset as large as $\pm 200 \mu \mathrm{m}$, albeit a slight decrease in the trench width (Figs. 4(d)-(f)). The second advantage of liquid immersion is the control over trench width by varying pulse energy. Owing to the focus offset tolerance, the variation of pulse energy does not significantly compromise the trench quality. Figure 5 shows an approximately linear relationship between pulse energy and trench width when performing micromachining in DI water 


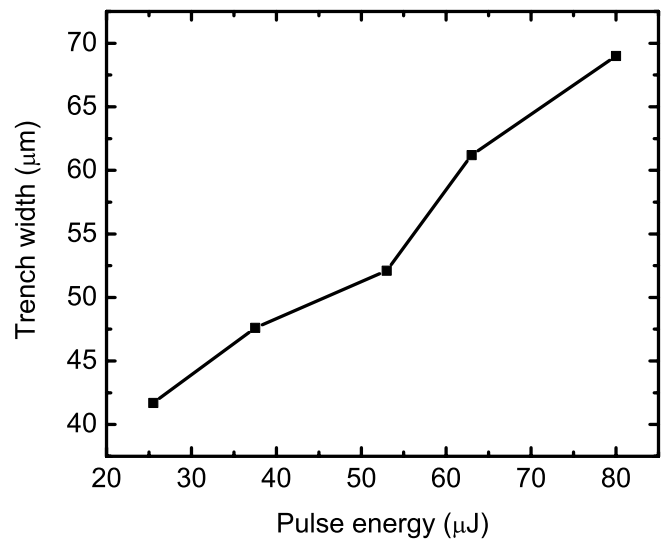

Fig. 5 Trench width control by varying pulse energy in liquid-immersion laser micromachining. The focus offset, pulse repetition rate and scan speed were fixed at $550 \mu \mathrm{m}, 1 \mathrm{kHz}$ and $25 \mu \mathrm{m} / \mathrm{s}$

Table 2 Density $\rho$, specific heat capacity $c$ and thermal conductivity $k$ of the substances involved in the experiment

\begin{tabular}{llll}
\hline & $\rho\left(\mathrm{g} \mathrm{cm}^{-3}\right)$ & $c\left(\mathrm{~J} \mathrm{~g}^{-1}{ }^{\circ} \mathrm{C}^{-1}\right)$ & $k\left(\mathrm{~W} \mathrm{~cm}^{-1}{ }^{\circ} \mathrm{C}^{-1}\right)$ \\
\hline $\mathrm{GaN}$ & 6.15 & 0.49 & 1.3 \\
$\mathrm{Al}_{2} \mathrm{O}_{3}$ & 4.025 & 0.75 & 0.35 \\
$\mathrm{H}_{2} \mathrm{O}$ & 1.0 & $4.18\left(\right.$ at $\left.25^{\circ} \mathrm{C}\right)$ & 0.006 \\
Air & $1.184 \times 10^{-3}$ & $1.012\left(\right.$ at $\left.23^{\circ} \mathrm{C}\right)$ & $2.5 \times 10^{-4}$ \\
\hline
\end{tabular}

at a fixed focus offset. For micromachining in ambient air, it is difficult to control the trench width just by altering the pulse energy. As the optimum focus offset depends strongly on the pulse energy, a new optimum focus offset needs to be determined when the pulse energy is altered by a small magnitude.

\section{Discussion}

The experimental results demonstrate that liquid immersion improves the trench micromachining process. It can be understood by taking a closer look on the laser-matter interaction for nanosecond-pulsed laser ablation in $\mathrm{GaN}$.

\subsection{Heat transfer}

Referring to Table 2, DI water has a much higher specific heat capacity and thermal conductivity than air. Therefore DI water is expected to carry the excess heat away from the irradiated region faster than air. To verify this, a simulation on the heat conduction process for single-pulse irradiation was performed. The heat equation (in cylindrical coordinates) was solved numerically by the finite element method (FEM):

$\rho c \frac{\partial T}{\partial t}-\nabla \cdot(k \nabla T)=Q$,

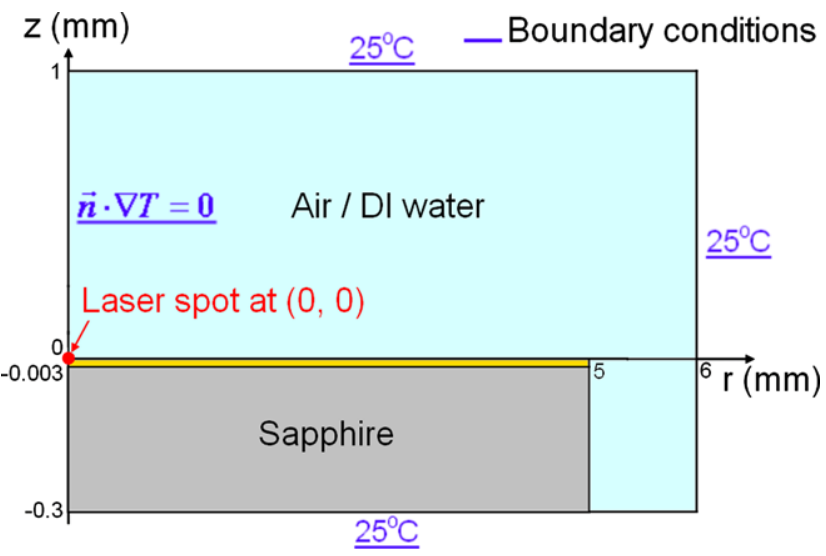

Fig. 6 Domain and boundary conditions for the heat transfer problem

where $\rho, c$ and $k$ are as defined in Table 2, $T$ is the temperature distribution $(\mathrm{K})$ and $Q$ presents the heat source $\left(\mathrm{W} \mathrm{mm}{ }^{-3}\right) . Q$ originates from the Gaussian laser beam, thus both $Q$ and the resulting $T$ are assumed to be radially symmetric. The domain and boundary conditions are defined in Fig. 6. The GaN and sapphire layers have thicknesses of $3 \mu \mathrm{m}$ and $300 \mu \mathrm{m}$, respectively. The Gaussian beam irradiates the $1 \mathrm{~cm} \times 1 \mathrm{~cm}$ sample at coordinates of $(r=0, z=0)$ towards the negative $z$-direction. Its intensity distribution is given by

$I(r, 0)=I_{0}\left(\frac{w_{0}^{2}}{w^{2}(0)}\right) \exp \left(\frac{-2 r^{2}}{w^{2}(0)}\right)$.

Here $I_{0}$ is the axial intensity at the beam waist, while $w_{0}$ and $w(0)$ are the $1 / \mathrm{e}^{2}$ radii at the beam waist and at $z=0$, respectively. Part of the incident laser energy is absorbed by $\mathrm{GaN}$ and gives rise to a heat source $Q$ in the following form:

$Q(r, z, t)=I(r, 0)(1-R) f(z) g(t)$,

where $R$ is the reflectivity of $\mathrm{GaN}(0.2077$ at $\lambda=349 \mathrm{~nm})$, and $I(r, 0)(1-R)$ is the absorbed intensity at the surface. $f(z)$ represents the attenuation of the laser beam as it penetrates into the GaN layer along the negative $z$ direction, which is modelled as

$f(z)= \begin{cases}\alpha \exp (\alpha z) & \text { if } z \leq 0 \\ 0 & \text { otherwise }\end{cases}$

where $\alpha$ is the absorption coefficient of $\mathrm{GaN}(111.9569 \times$ $10^{3} \mathrm{~cm}^{-1}$ at $\lambda=349 \mathrm{~nm}$ ). The last function $g(t)$ is used for pulse shaping, which is assumed to take the following form:

$g(t)=\frac{\tau_{\mathrm{FWHM}} t}{\tau_{r}^{2} \mathrm{e}} \exp \left(1-\frac{t}{\tau_{r}}\right)$,

where $\tau_{r}$ is the pulse rise time. For a $\tau_{\mathrm{FWHM}}$ of $4 \mathrm{~ns}, \tau_{r}$ is estimated to be $1.6351 \mathrm{~ns}$. The parameters $I_{0}, w_{0}$ and $w(0)$ 
are estimated by the formulae below:

$f_{\text {eff }} \approx f+(n-1) h$,

$w_{0}=(2 \lambda / \pi)\left(f_{\text {eff }} / D\right)$,

$w(0)=w_{0} \sqrt{1+\left(\lambda \Delta z / \pi w_{0}^{2}\right)^{2}}$,

$I_{0}=2 E_{p} /\left(\pi w_{0}^{2} \tau_{\mathrm{FWHM}}\right)$,

where $f$ is the focal length of the objective lens $(19 \mathrm{~mm})$, $n$ is the refractive index of water, $h$ is the height of water above the sample $(1 \mathrm{~mm}), f_{\text {eff }}$ is the effective focal length, $D$ is the collimated beam width $(2.5 \mathrm{~mm}), E_{p}$ is the pulse energy $(25 \mu \mathrm{J})$, and $\Delta z$ is the focus offset $(400 \mu \mathrm{m})$. We take $f(z)$ and $g(t)$ to be the expressions above because the integration of $Q$ over the spatial domain where $z \leq 0$ and the whole temporal domain gives the absorbed portion of pulse energy $E_{p}(1-R)$. With this set of formulae and data, the temperature distribution $T$ can be solved. Figure 7 shows the variation of $\mathrm{GaN}$ surface temperature during the initial $100 \mathrm{~ns}$ at the centre of the laser spot $(r=0, z=0)$ (solid curves) and near the trench edge $(r=0.015, z=0)$ (dotted

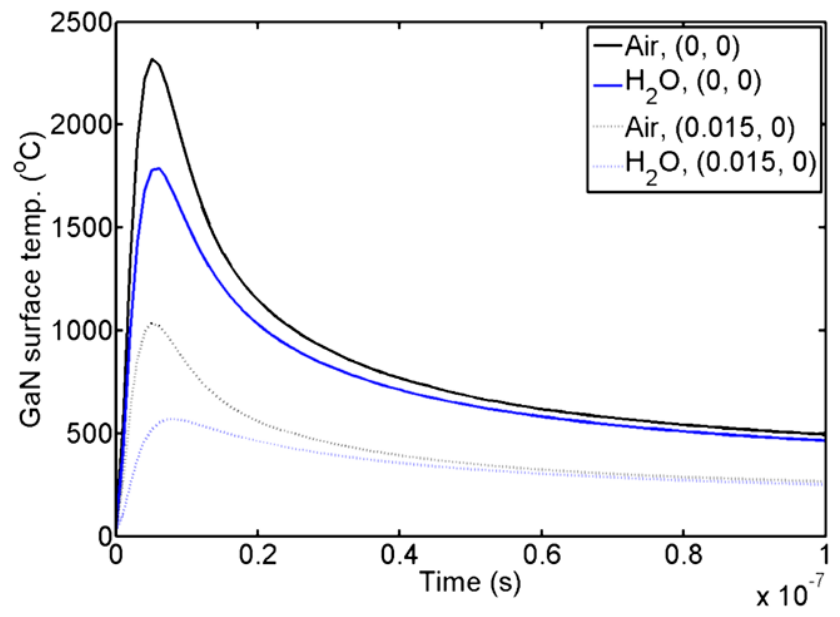

Fig. 7 Temporal variation of GaN surface temperature at two different positions: at the centre of laser spot (solid); near the edge of the trench (dotted) curves). It is observed that the GaN surface temperature in air is always higher than that in DI water at both positions. In addition, sharper temperature peaks are found when the micromachining is performed in air. The maximum temperature is as high as $1000^{\circ} \mathrm{C}$ even near the trench edge. It is known that $\mathrm{GaN}$ begins to decompose into liquid $\mathrm{Ga}$ and $\mathrm{N}_{2}$ gas at a temperature of $900^{\circ} \mathrm{C}$ [16]. The rapid heating and cooling cycles in air can result in the increased generation and resolidification of molten $\mathrm{Ga}$ within each pulse period. The resolidified Ga droplets deposit around the sidewall, degrading the surface quality. Another consequence of rapid heating and cooling is the increased thermal stress incurred in the crystal structure of GaN. Cracks may be generated as a result.

The two-dimensional temperature distribution also demonstrates the strong cooling effect of water. As shown in Figs. 8(a) and (b), at $t=1 \mu \mathrm{s}$, the temperature of water immediately above the sample surface is lower than that of air. The slight decrease of water temperature below the room temperature can be attributed to the adiabatic expansion of water vapour as well as the vapour plume of ablated material [17]. At this instance, the GaN surface temperature in water is in general lower than that in air, indicating efficient heat extraction by water. The heat-affected zone (HAZ) is also seen to be smaller when the sample is immersed in DI water.

\subsection{Plasma-induced recoil pressure}

High-energy laser pulses are able to melt, vaporize and ionize the material being irradiated, resulting in the formation of plasma. When the plasma expands, a recoil pressure is exerted on the sample surface, causing material removal. If the ambient medium is water instead of air, the expansion of plasma will be confined by the underwater pressure. This leads to an increase in the plasma-induced recoil pressure. The following theoretical calculation verifies this statement. By treating air as an ideal gas, the recoil pressure $P$ (kbar)
Fig. 8 (a) and (b): Temperature distribution at $t=1 \mu \mathrm{s}$ when the ambient medium is: (a) air; (b) DI water
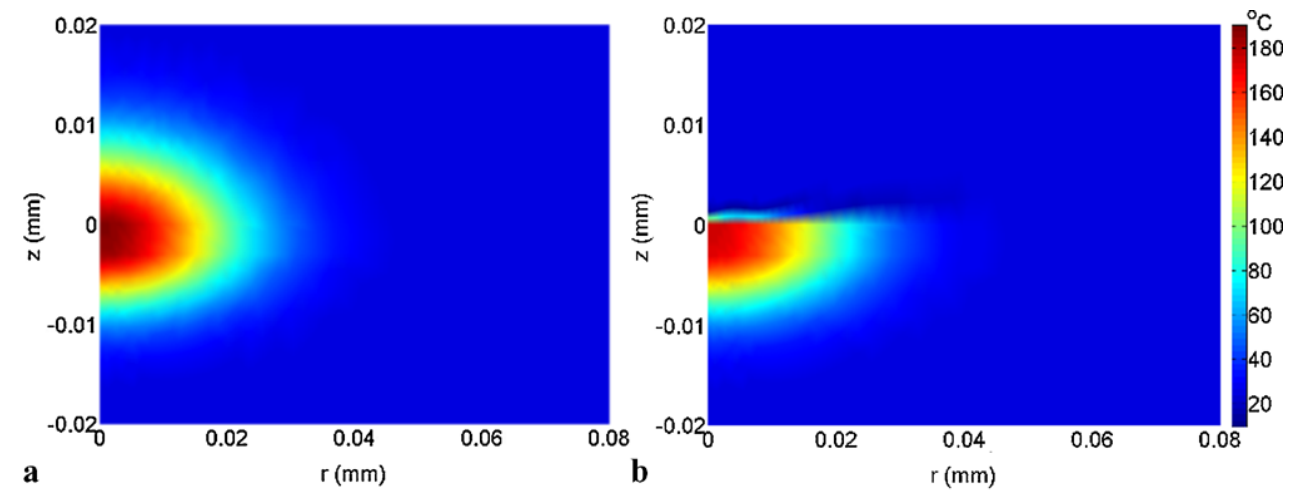
in ambient air can be estimated by [18]:

$P=32.2\left(\frac{a}{2 a+3}\right)^{2 / 3}(4 \rho)^{1 / 3} I_{a}^{2 / 3}$.

Here, $a$ is the ratio between thermal energy and internal energy, which is equal to 1 for ideal gas. $\rho\left(\mathrm{g} / \mathrm{cm}^{3}\right)$ is the density of air. $I_{a}\left(\mathrm{GW} / \mathrm{cm}^{2}\right)$ is the absorbed intensity of the laser beam, which is assumed to be constant in [18]. Taking $I_{a}=I(0,0)(1-R)=0.4652 \mathrm{GW} / \mathrm{cm}^{2}, P$ is found to be $1.11 \mathrm{kbar}$. If the sample is immersed into water, the recoil pressure is determined by another formula [19]:

$P=0.1 \sqrt{\frac{a}{2 a+3} I_{a} Z}$

where $2 / Z=1 / Z_{\text {water }}+1 / Z_{\mathrm{GaN}}$. $Z_{\text {water }}$ and $Z_{\mathrm{GaN}}$ are the shock impedances of water and GaN, which is given by the product of the density and speed of sound of the respective material. With $Z_{\text {water }}=1.65 \times 10^{5} \mathrm{~g} \mathrm{~cm}^{-2} \mathrm{~s}^{-1}$, $Z_{\mathrm{GaN}}=4.92 \times 10^{6} \mathrm{~g} \mathrm{~cm}^{-2} \mathrm{~s}^{-1}$ and $a=0.2$ for water, $P$ is estimated to be $9.35 \mathrm{kbar}$, which is 8.4 times greater than that in air. This tremendous increase of recoil pressure facilitates the liquid-phase expulsion of Ga from the ablated region, making the trench free of redeposited material.

\subsection{Plasma shielding effect in water}

The plasma generated from laser ablation can absorb part of the incident laser energy and reduce the energy delivered to the sample. This is known as plasma shielding effect. The effect becomes stronger when the plasma has a larger size, longer duration and a starting time that overlaps with the laser pulse. Because of water confinement effects, the plasma size and duration are much reduced in water. The onset of plasma formation is also delayed by $5 \mathrm{~ns}$, reducing the overlap between the laser pulse and the plasma [20]. These factors weaken the plasma shielding effect, causing a stronger coupling of the laser beam with the material. Material removal is thus more efficient in water.

\subsection{Collapse of cavitation bubbles}

Formation of cavitation bubbles is a process that happens solely in liquids. In our experiment, the bubbles may originate from the dissolved gases in DI water, or the $\mathrm{N}_{2}$ gas from the laser ablation of GaN. When a bubble collapses near the sample surface, a high-speed liquid jet directed towards the surface will be generated. If there is no water between the bubble and sample surface, the liquid jet will produce a strong impulse towards the sample. This impulse can be 5.2-12.4 times that of the laser ablation impact in air [13], which further helps the liquid-phase expulsion of Ga.

\section{Conclusion}

Nanosecond-laser micromachining for trench formation on $\mathrm{GaN} /$ sapphire wafer in ambient DI water has been demonstrated. Comparing with ambient air, DI water offers the following three advantages: smoother and cleaner ablated surfaces, focus offset tolerance and control of trench width by pulse energy. The quality of trenches is even comparable to those produced by femtosecond-laser micromachining in air. The reasons behind such improvement include: enhanced heat transfer due to the higher heat capacity and thermal conductivity of water, increased plasma-induced recoil pressure, weakened plasma shielding effect and the collapse of cavitation bubbles. All these factors contribute to the efficient removal of molten $\mathrm{Ga}$ and reduction of its redeposition in the vicinity of the trenches. This technique can be applied to the rapid prototyping of GaN-based devices.

Acknowledgement This work was supported by a GRF grant of the Research Grant Council of Hong Kong (project HKU 7118/09E).

Open Access This article is distributed under the terms of the Creative Commons Attribution Noncommercial License which permits any noncommercial use, distribution, and reproduction in any medium, provided the original author(s) and source are credited.

\section{References}

1. E. Gu, C.W. Jeon, H.W. Choi, G. Rice, M.D. Dawson, E.K. Illy, M.R.H. Knowles, Thin Solid Films 453-454, 462 (2004)

2. X.H. Wang, P.T. Lai, H.W. Choi, J. Vac. Sci. Technol., B Microelectron. Process. Phenom. 27, 1048 (2009)

3. T. Fujii, Y. Gao, R. Sharma, E.L. Hu, S.P. DenBaars, S. Nakamura, Appl. Phys. Lett. 84, 855 (2004)

4. E. Gu, H. Howard, A. Conneely, G.M. O'Connor, E.K. Illy, M.R.H. Knowles, P.R. Edwards, R.W. Martin, I.M. Watson, M.D. Dawson, Appl. Surf. Sci. 252, 4897 (2006)

5. K. Ozono, M. Obara, A. Usui, H. Sunakawa, Opt. Commun. 189, $103(2001)$

6. G.Y. Mak, E.Y. Lam, H.W. Choi, J. Vac. Sci. Technol., B Microelectron. Process. Phenom. 28, 380 (2010)

7. T. Akane, K. Sugioka, K. Midorikawa, Appl. Phys. A 69, S309 (1999)

8. X. Li, T. Jia, D. Feng, Z. Xu, Appl. Surf. Sci. 225, 339 (2004)

9. J.F. Muth, J.H. Lee, I.K. Shmagin, R.M. Kolbas, H.C. Casey, B.P. Keller, U.K. Mishra, S.P. DenBaars, Appl. Phys. Lett. 71, 2572 (1997)

10. B.S. Patel, Z.H. Zaidi, Meas. Sci. Technol. 10, 146 (1999)

11. C.-H. Tsai, C.-C. Li, J. Mater. Process. Technol. 209, 2838 (2009)

12. A.K. Nath, D. Hansdah, S. Roy, A.R. Choudhury, J. Appl. Phys. 107, 123103 (2010)

13. J. Lu, R.Q. Xu, X. Chen, Z.H. Shen, X.W. Ni, S.Y. Zhang, C.M. Cao, J. Appl. Phys. 95, 3890 (2004)

14. C.D. Marco, S.M. Eaton, R. Suriano, S. Turri, M. Levi, R. Ramponi, G. Cerullo, R. Osellame, Appl. Mater. \& Interfaces 2, 2377 (2010) 
15. O. Ambacher, M.S. Brandt, R. Dimitrov, T. Metzger, M. Stutzmann, R.A. Fischer, A. Miehr, A. Bergmaier, G. Dollinger, J. Vac. Sci. Technol., B Microelectron. Process. Phenom. 14, 3532 (1996)

16. H.W. Choi, M.A. Rana, S.J. Chua, T. Osipowicz, J.S. Pan, Semicond. Sci. Technol. 17, 1223 (2002)

17. A.V. Gusarov, A.G. Gnedovets, I. Smurov, Appl. Surf. Sci. 154155, 66 (2000)
18. R. Fabbro, J. Fournier, P. Ballard, D. Devaux, J. Virmont, J. Appl. Phys. 68, 775 (1990)

19. D. Devaux, R. Fabbro, L. Tollier, E. Bartnicki, J. Appl. Phys. 74, 2268 (1993)

20. M.H. Hong, M.L. Koh, S. Zhu, Y.F. Lu, T.C. Chong, Appl. Surf. Sci. 197-198, 911 (2002) 